\title{
DOENÇA RENAL CRÔNICA: FREQUENTE E GRAVE, MAS TAMBÉM PREVENÍVEL E TRATÁVEL
}

\author{
Marcus Gomes Bastos ${ }^{1 *}$, Rachel Bregman², Gianna Mastroianni Kirsztajn ${ }^{3}$ \\ Trabalho realizado nas Universidades - Universidade Federal de Juiz de Fora - MG, Universidade do Estado do Rio de Janeiro - UERJ e Universidade Federal de \\ São Paulo - UNIFESP, São Paulo, SP
}

\author{
*Correspondência: \\ NIEPEN da Universidade \\ Federal de Juiz de Fora - \\ UFJF e Fundação IMEPEN \\ Rua José Lourenço Kelmer \\ $n^{\circ} 1300$ \\ São Pedro \\ Juiz de Fora - MG \\ CEP: 36036-330
}

\begin{abstract}
RESUMO
A doença renal crônica é considerada problema de saúde pública em todo o mundo. No Brasil, a incidência e a prevalência de falência de função renal estão aumentando; o prognóstico ainda é ruim e os custos do tratamento da doença são altíssimos. Independentemente da etiologia da doença de base, os principais desfechos em pacientes com DRC são as suas complicações (anemia, acidose metabólica, desnutrição e alteração do metabolismo de cálcio e fósforo), decorrentes da perda funcional renal, óbito (principalmente, por causas cardiovasculares) e perda de função renal. Estudos recentes indicam que estes desfechos indesejados podem ser prevenidos ou retardados se a DRC for diagnosticada precocemente e as medidas nefro e cardioprotetoras implementadas o mais rápido possível. $\mathrm{O}$ atual estagiamento da doença e uma descrição dessas medidas preventivas são apresentados na presente revisão.
\end{abstract}

Unitermos: Falência renal crônica. Proteinúria. Albuminúria. Taxa de filtração glomerular. Anemia. Diabetes mellitus.

\section{INTRODUÇÃO}

A nova definição da doença renal crônica (DRC), em uso desde 2002, propiciou um estagiamento da doença que independe da sua causa. A partir desta nova abordagem, ficou evidente que a DRC é muito mais frequente do que até então se considerava e sua evolução clínica está associada a taxas altas de morbimortalidade. ${ }^{1}$

Os rins são órgãos fundamentais para a manutenção da homeostase do corpo humano. Assim, não é surpresa constatarmos que, diminuição progressiva da função renal, implique em comprometimento de essencialmente todos os outros órgãos. A função renal é avaliada pela filtração glomerular (FG) e a sua diminuição é observada na Doença Renal Crônica (DRC), associada a perda das funções regulatórias, excretórias e endócrinas do rim. Quando a FG atinge valores muito baixos, inferiores a $15 \mathrm{~mL} / \mathrm{min} / 1,73 \mathrm{~m}^{2}$, estabelece-se o que denominamos falência funcional renal (FFR), ou seja, o estágio mais avançado do continuum de perda funcional progressiva observado na DRC.

A DRC é, atualmente, considerada um problema de saúde pública mundial. No Brasil, a incidência e a prevalência de FFR estão aumentando, o prognóstico ainda é ruim e os custos do tratamento da doença são altíssimos. O número projetado atualmente para pacientes em tratamento dialítico e com transplante renal no Brasil está próximo dos 120.000, a um custo de 1,4 bilhão de reais. ${ }^{2}$ Independentemente da doença de base, os principais desfechos em pacientes com DRC são as suas complicações (anemia, acidose metabólica, alteração do metabolismo mineral e desnutrição), decorrentes da perda funcional renal, óbito (principalmente por causas cardiovasculares) e FFR. Estudos recentes indicam que estes desfechos indesejados podem ser prevenidos ou retardados se a DRC for diagnosticada precocemente e as medidas nefro e cardioprotetoras implementadas precocemente. ${ }^{1,3-5}$ Infelizmente, a DRC é subdiagnosticada e tratada inadequadamente, resultando na perda de oportunidade para a implementação de prevenção primária, secundária e terciária, em parte devido à falta de conhecimento da definição e classificação dos estágios da doença, bem como a não utilização de testes simples para o diagnóstico e avaliação funcional da doença.

1. Doutorado em Nefrologia - Professor de Nefrologia e coordenador do Núcleo Interdisciplinar de Estudos, Pesquisas e Tratamento em Nefrologia - (NIEPEN) da Universidade Federal de Juiz de Fora - (UFJF); editor do Jornal Brasileiro de Nefrologia e membro do Comitê de Prevenção de Doença Renal Crônica da Sociedade Brasileira de Nefrologia, Juiz de Fora, MG

2. Doutorado em Nefrologia - Professora adjunta da disciplina de Nefrologia da Universidade do Estado do Rio de Janeiro; membro do Comitê de Doença Renal Crônica da Sociedade Brasileira de Nefrologia e membro do Comitê de Anemia da Sociedade Latino Americana de Nefrologia e Hipertensão, Rio de Janeiro, RJ

3. Doutorado em Nefrologia - Livre-Docente - Médica coordenadora do Setor de Glomerulopatias da Universidade Federal de São Paulo - (UNIFESP); coordenadora do Comitê de Prevenção de Doença Renal Crônica da Sociedade Brasileira de Nefrologia, São Paulo, SP 


\begin{tabular}{lll}
\hline \multicolumn{3}{c}{ Tabela 1 - Estagiamento da DRC proposta pelo grupo de } \\
trabalho do K/DOQI \\
\hline Estágio & Descrição & FG* $^{*}$ \\
\hline 1 & Lesão renal com FG normal ou aumentada & $\geq 90$ \\
2 & Lesão renal com FG levemente diminuída & $60-89$ \\
3 & Lesão renal com FG moderadamente & $30-59$ \\
& diminuída \\
5 & Lesão renal com FG severamente diminuída & $15-29$ \\
5 & FFR & \\
& substitutiva & $<15$ \\
\end{tabular}

*FG = Filtração glomerular em $\mathrm{mL} / \mathrm{min} / 1,73 \mathrm{~m}^{2 ; * *} \mathrm{FFR}=$ Falência funcional renal

Vale salientar que a maioria das diretrizes definem a FFR como: 1) FG menor que $15 \mathrm{~mL}$ $\mathrm{min} / 1,73 \mathrm{~m}^{2}$, a qual é acompanhada frequentemente por sinais e sintomas de uremia ou 2 a necessidade de início de terapia de substituição da função renal (diálise ou transplante). ${ }^{1,3-5}$

\section{Conceito de Doença Renal Crônica (DRC) ${ }^{1,3-5}$}

Recentemente, a Sociedade Brasileira de Nefrologia ${ }^{3}$ referendou a definição de DRC proposta pela National Kidney Foundation Americana (NKF), em seu documento Kidney Disease Outcomes Quality Initiative (K/DOQI), que se baseia nos seguintes critérios: ${ }^{1}$

- Lesão presente por um período igual ou superior a três meses, definida por anormalidades estruturais ou funcionais do rim, com ou sem diminuição da FG, evidenciada por anormalidades histopatológicas ou de marcadores de lesão renal, incluindo alterações sanguíneas ou urinárias, ou ainda de exames de imagem;

- $\mathrm{FG}<60 \mathrm{~mL} / \mathrm{min} / 1,73 \mathrm{~m}^{2}$ por um período igual ou superior a três meses com ou sem lesão renal.

Assim, a DRC é definida pela lesão do parênquima renal (com função renal normal) e/ou pela diminuição funcional renal presentes por um período igual ou superior a três meses. Com base nesta definição, foi proposta a classificação (estagiamento) para a DRC ${ }^{1}$, apresentada na Tabela 1.

\section{Grupos de risco para DRC ${ }^{1}$}

Alguns pacientes apresentam suscetibilidade aumentada para DRC e são considerados grupos de risco. São eles:

1. Hipertensos: A hipertensão arterial é comum na DRC, podendo ocorrer em mais de $75 \%$ dos pacientes de qualquer idade;

2. Diabéticos: Os pacientes diabéticos apresentam risco aumentado para DRC e doença cardiovascular e devem ser monitorizados frequentemente para a ocorrência da lesão renal;

3. Idosos: A diminuição fisiológica da FG e, as lesões renais que ocorrem com a idade, secundárias a doenças crônicas comuns em pacientes de idade avançada, tornam os idosos susceptíveis a DRC;

4. Pacientes com doença cardiovascular (DCV): A DRC é considerada fator de risco para DCV e estudo recente demonstrou que a DCV se associa independentemente com diminuição da FG e com a ocorrência de DRC;

5. Familiares de pacientes portadores de DRC: Os familiares de pacientes portadores de DRC apresentam prevalência aumentada de hipertensão arterial, Diabetes mellitus, proteinúria e doença renal.
6. Pacientes em uso de medicações nefrotóxicas: O uso de medicações nefrotóxicas deveria ser evitado ou otimizado nos pacientes com DRC, particularmente quando a FG é menor que $60 \mathrm{~mL} / \mathrm{min} / 1,73 \mathrm{~m}^{2}$.

\section{Diagnóstico da DRC}

\section{Avaliação da Filtração Glomerular}

A FG é a melhor medida do funcionamento renal em indivíduos normais ou pacientes com doença renal. ${ }^{1,3-6} \mathrm{O}$ nível da FG varia com a idade, sexo, e massa muscular. A FG diminui com a idade. FG menor que $60 \mathrm{~mL} / \mathrm{min} / 1,73 \mathrm{~m}^{2}$ representa diminuição de cerca de $50 \%$ da função renal normal e, abaixo deste nível, aumenta a prevalência das complicações da DRC. ${ }^{6-8}$

Embora a diminuição da FG relacionada à idade possa dever-se ao processo de envelhecimento normal, a diminuição da FG no idoso é um preditor independente de evolução adversa da doença, tal como óbito e doença cardiovascular. ${ }^{9-11}$ Além do mais, a FG diminuída no idoso deve implicar em ajustes nas dosagens das medicações, semelhantemente ao que se faz em todos os pacientes com DRC. Assim, a definição da DRC é a mesma, independente da idade. Como a FG diminui com a idade, a prevalência de DRC aumenta nos pacientes idosos; cerca de $17 \%$ das pessoas com mais de 60 anos apresentam FG menor que $60 \mathrm{~mL} / \mathrm{min} / 1,73 \mathrm{~m}^{2} .{ }^{12}$

A FG, idealmente, deveria ser determinada pela depuração da inulina ou de materiais radioisotópicos (p. ex., DTPA). Infelizmente, tais métodos, além de onerosos, não são de fácil utilização no dia a dia. Na prática clínica, a FG pode ser determinada pela dosagem da creatinina sérica e/ou pela depuração desta pelo rim. A depuração da creatinina pode ser realizada em urina coletada no período de 24 horas, porém a coleta urinária inadequada, seja por falta de compreensão do procedimento ou tipo de atividade do paciente, é um limitador do método. ${ }^{2}$

Mais recentemente, as diretrizes preconizam que a FG pode ser estimada a partir da dosagem sérica da creatinina ( $\mathrm{Crs}$ ), aliada a variáveis demográficas, tais como: idade, sexo, raça e tamanho corporal. As duas equações mais frequentemente utilizadas encontram-se a seguir:

1. Equação de Cockcroft-Gault: ${ }^{13}$

Depuração de creatinina $(\mathrm{mL} / \mathrm{min})=140$ - idade (em anos) $x$ peso (quilogramas) $/ 72 \times \mathrm{Crs}$ ( $\mathrm{x} 0,85$ se mulher)

2. Equação abreviada do estudo Modification of Diet in Renal Disease (MDRD) ${ }^{14}$

Filtração glomerular $\left(\mathrm{mL} / \mathrm{min} / 1,73 \mathrm{~m}^{2}\right)=186 \times(\mathrm{Crs})^{-1,154}$ $x$ (idade) $)^{-0,203} \times(0,742$ se mulher $) \times(1,210$ se indivíduo afro-americano)

A fórmula de Cockcroft-Gault e a utilizada no estudo MDRD apresentam excelente correlação com a determinação da FG avaliada com DTPA e já foram amplamente empregadas em vários estudos em diferentes partes do mundo, inclusive no Brasil. ${ }^{15}$ Atualmente, as fórmulas que estimam a FG estão disponibilizadas em programas para computadores manuais, nas páginas da internet da Sociedade Brasileira de Nefrologia e na National Kidney Foundation ou na forma de tabelas. ${ }^{16}$

\section{Avaliação da lesão do parênquima renal}

A DRC pode ser diagnosticada sem o conhecimento da sua causa. ${ }^{1}$ Geralmente, o comprometimento do parênquima renal 


\section{Figura - Avaliação da proteinúria em indivíduos de grupo de risco e sem doença renal evidente}

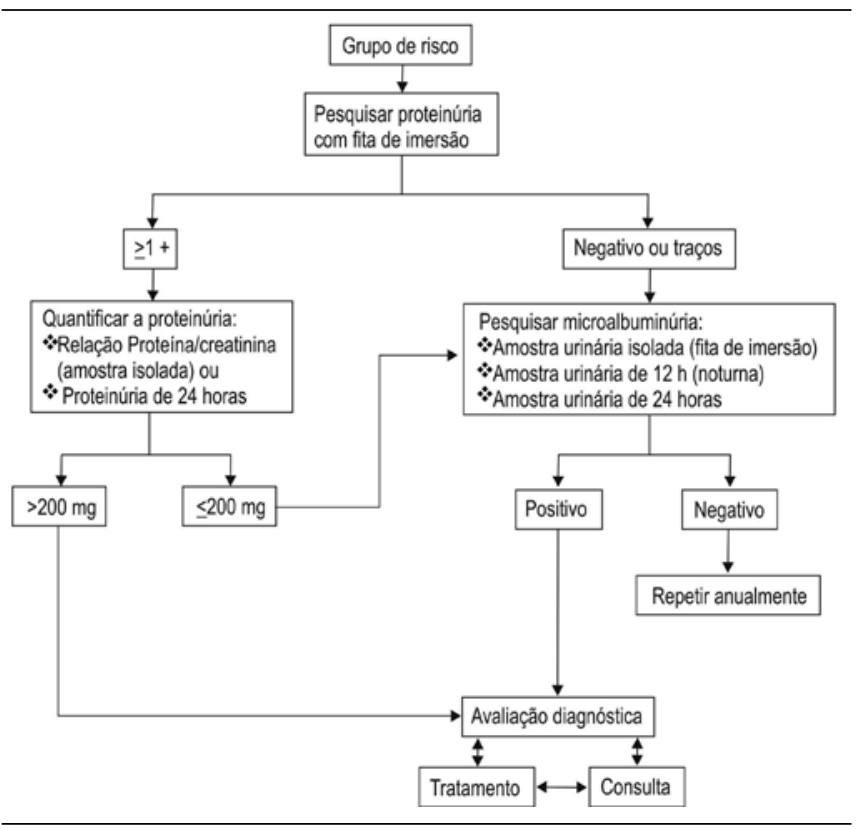

é confirmado por marcadores de lesão em vez da biópsia renal. De acordo com as Diretrizes do $\mathrm{K}_{\mathrm{DDOQ}}{ }^{1}, \mathrm{SBN}^{3}$, inglesas ${ }^{4} \mathrm{e}$ canadenses ${ }^{5}$, a proteinúria (albuminúria) persistente é o principal marcador de lesão renal. A Tabela 2 sumariza os valores de albuminúria recomendados para o diagnóstico da DRC. Outros marcadores de lesão renal incluem anormalidades no sedimento urinário (principalmente hematúria e leucocitúria), alterações de parâmetros bioquímicos no sangue e na urina e alterações nos exames de imagem. ${ }^{1}$ Pacientes com FG normal, mas com marcador(es) de lesão renal (particularmente albuminúria) apresentam risco aumentado para evolução da DRC. ${ }^{18}$

Indivíduos normais excretam pequena quantidade de proteína na urina diariamente, numa faixa considerada como fisiológica. No entanto, a excreção de quantidade aumentada de proteína na urina é um marcador sensível para DRC secundária a diabetes (doença renal diabética), glomerulopatias primárias e secundárias e hipertensão arterial. Já as proteínas de baixo peso molecular, quando em quantidade anormal na urina, sugerem a ocorrência de doenças túbulo-intersticiais. ${ }^{1,3}$

É preciso deixar claro que proteinúria é um termo genérico que engloba a excreção urinária de albumina e qualquer outro tipo de proteína. Já a palavra albuminúria refere-se única e exclusivamente à eliminação urinária de albumina, um marcador de lesão glomerular. Além disso, o termo microalbuminúria é utilizado quando a quantidade de albumina urinária está acima do normal, porém aquém dos níveis detectados nos testes de proteinúria total. Os valores que definem tais condições encontram-se na Tabela 2.1,4

A figura apresentada é uma proposta de algoritmo para pesquisar proteinúria como marcador de lesão renal em indivíduos que compõem grupos de risco e sem evidências de doença renal. 0 primeiro passo é pesquisar a proteinúria com fita de imersão numa amostra urinária isolada. A ocorrência de uma ou mais cruzes de proteinúria determina a necessidade de quantificação, que pode ser feita em amostra urinária isolada (relação proteína/creatinina) ou na urina de 24 horas. Valores superiores a $200 \mathrm{mg}$ implicam em avaliação diagnóstica e tratamento. Se o paciente faz parte de grupo de risco para DRC e a fita de imersão resultar negativa, o próximo passo é pesquisar microalbuminúria em amostra urinária isolada (com fita de imersão, método semiquantitativo) ou urina de 12 horas noturna ou em amostra urinária de 24 horas. Se o resultado for negativo, repetir a avaliação anualmente. Em caso de resultado positivo, ou seja, dois ou mais resultados positivos em intervalo superior a três meses entre os testes, deve-se aprofundar a investigação, objetivando confirmação diagnóstica e instituição de tratamento nefroprotetor.

\section{Tratamento}

O tratamento dos pacientes com DRC requer o reconhecimento de aspectos distintos, porém relacionados, que englobam a doença de base, o estágio da doença, a velocidade da diminuição da FG, identificação de complicações e comorbidades, particularmente as cardiovasculares. Seguem algumas orientações:

\begin{tabular}{|c|c|c|c|c|}
\hline & $\begin{array}{l}\text { Método de coleta } \\
\text { de urina }\end{array}$ & Normal & $\begin{array}{c}\text { Micro- } \\
\text { Albuminúria } \\
\end{array}$ & $\begin{array}{c}\text { Albuminúria ou Proteinúria } \\
\text { Clínica }\end{array}$ \\
\hline \multirow{3}{*}{$\begin{array}{l}\text { Proteína } \\
\text { total }\end{array}$} & Urina de 24 horas & $\leq 300 \mathrm{mg} / \mathrm{dia}$ & Não aplicável & $>300 \mathrm{mg} / \mathrm{dia}$ \\
\hline & $\begin{array}{l}\text { Urina isolada (fita de } \\
\text { imersão) }\end{array}$ & $\leq 30 \mathrm{mg} / \mathrm{dL}$ & Não aplicável & $>30 \mathrm{mg} / \mathrm{dL}$ \\
\hline & $\begin{array}{l}\text { Urina isolada (relação } \\
\text { proteína/creatinina) }\end{array}$ & $\leq 200 \mathrm{mg} / \mathrm{g}$ & Não aplicável & >200 mg/g \\
\hline \multirow[t]{2}{*}{ Albumina } & $\begin{array}{l}\text { Urina de } 24 \text { horas } \\
\text { Urina isolada (fita de } \\
\text { imersão específica para } \\
\text { albumina) }\end{array}$ & $\begin{array}{l}\leq 30 \mathrm{mg} / \mathrm{dL} \\
\leq 3 \mathrm{mg} / \mathrm{dL}\end{array}$ & $\begin{array}{c}30-300 \mathrm{mg} / \mathrm{dia} \\
\quad>3 \mathrm{mg} / \mathrm{dL}\end{array}$ & $\begin{array}{l}\text { >300 mg/dia } \\
\text { Não aplicável }\end{array}$ \\
\hline & $\begin{array}{l}\text { Urina isolada (relação } \\
\text { albumina/creatinina) }\end{array}$ & $\begin{array}{l}<17 \mathrm{mg} / \mathrm{g}\left(\mathrm{M}^{*}\right) \\
<25 \mathrm{mg} / \mathrm{g}\left(\mathrm{H}^{* *}\right)\end{array}$ & $\begin{array}{l}17-250 \mathrm{mg} / \mathrm{g}(\mathrm{M}) \\
25-355 \mathrm{mg} / \mathrm{g}(\mathrm{H})\end{array}$ & $\begin{array}{l}>250 \mathrm{mg} / \mathrm{g}(\mathrm{M}) \\
>355 \mathrm{mg} / \mathrm{g} \mathrm{(H)}\end{array}$ \\
\hline
\end{tabular}


Hipertensão arterial: A hipertensão é uma causa frequente de DRC. ${ }^{19}$ A transmissão da hipertensão sistêmica para o glomérulo determina lesão no capilar glomerular. ${ }^{20-21}$ Alguns aspectos devem ser observados no tratamento da hipertensão arterial: ${ }^{1,3-5}$

- Pacientes com DRC, cursando com proteinúria $>1,0 \mathrm{~g} /$ dia, devem ser tratados preferencialmente com inibidores da enzima de conversão da angiotensina (IECA) ou, em caso de intolerância a este grupo de drogas, com bloqueadores do receptor 1 da angiotensina (BRAT 1). O nível pressórico recomendado é < 125/75 mmHg;

- Pacientes com DRC, cursando com proteinúria $<1,0$ g/dia, recomenda-se o tratamento com IECA, BRAT 1 . O nível pressórico recomendado é < 130/80 $\mathrm{mmHg}$;

- Para pacientes hipertensos, com DRC associada à doença vascular da artéria renal, o tratamento deve ser semelhante ao dos pacientes hipertensos.

Recomenda-se precaução quando se utilizar IECA ou BRAT 1 pelo risco de insuficiência renal aguda e/ou hiperpotassemia. Importante avaliar se a lesão da artéria renal é uni ou bilateral.

Os pacientes que necessitarem de maior número de drogas para o controle pressórico devem ser tratados da mesma foram que a população geral.

Proteinúria: A proteinúria merece destaque especial, pois além de ser um excelente marcador de lesão renal é um importante fator de risco para progressão da DRC, bem como para morbimortalidade cardiovascular. ${ }^{18,22}$ A proteinúria é considerada persistente quando presente em pelo menos duas de três avaliações urinárias. As medidas terapêuticas correspondem a: $2,3,4,5$

- Os IECAS e os BRAT 1 são as medicações de eleição para a redução da albuminúria. Pacientes com diabetes e albuminúria persistente devem ser tratados com um destes grupos de drogas para retardar a progressão da DRC.

- Em pacientes selecionados, é possível diminuir a albuminúria com os antagonistas do receptor da aldosterona.

- Medidas adicionais: controle da ingestão excessiva de proteínas e redução do peso corporal (para pacientes com Índice de Massa Corporal acima do normal).

Anemia: A anemia, definida como níveis de hemoglobina $<13,0 \mathrm{~g} / \mathrm{dL}$ no homem e $<12,0 \mathrm{~g} / \mathrm{dL}$ na mulher, é uma das complicações mais frequentes e precoces no curso da DRC. A anemia se associa com evolução adversa da DRC, incluindo hospitalização, doença cardiovascular, mortalidade e diminuição da qualidade de vida dos pacientes. ${ }^{23}$ A principal causa de anemia na DRC é a deficiência de produção da eritropoietina pelos fibroblastos peritubulares renais. ${ }^{24}$ Contudo, é importante mencionar que outros fatores podem determinar a ocorrência de anemia nesses pacientes, particularmente a deficiência de ferro. ${ }^{25}$ No momento, não existem evidências da associação entre normalização da hemoglobina e melhor prognóstico nos pacientes com DRC. A recomendação atual é manter os níveis de hemoglobina entre 11,0-12,0 g/dL para pacientes com DRC e anemia. ${ }^{26,27}$ É fundamental que o paciente tenha estoque normal de ferro para se iniciar o tratamento com eritropoietina. Este deve ser administrado para manter o índice de saturação da transferrina $>20 \%$ e a ferritina $>100 \mathrm{ng} / \mathrm{dL} .{ }^{26,27} \mathrm{O}$ uso de eritropoietina em pacientes com DRC pode associar-se a efeitos colaterais como hipertensão arterial e complicações trombóticas, particularmente quando as doses semanais ultrapassam 15.000 UI. ${ }^{28}$

Alterações do metabolismo mineral: A função renal normal é fundamental na manutenção do balanço do cálcio e do fósforo. A diminuição da FG se associa com hiperfosfatemia e hipocalcemia, alterações que exercem papel fisiopatológico na doença óssea e calcificação vascular nesses pacientes. Com a perda da capacidade funcional renal, diminui a $1 \alpha$-hidroxilação da 25-hidroxivitamina $\mathrm{D}$ e, consequentemente, a síntese renal de 1,25-dihidroxivitamina $\mathrm{D}$ (calcitriol), forma ativa da vitamina D. A deficiência da produção de calcitriol determina hiperplasia das glândulas paratireoides, seguida de hiperparatireoidismo secundário. ${ }^{29} \mathrm{~A}$ monitorização do cálcio, do fósforo e do paratohormônio (PTH) deve ser realizada obrigatoriamente em todos os pacientes com DRC estágios 4 e 5 e nos pacientes no estágio 3 que apresentam perda progressiva da FG. $2,3,4,5,29$

Acidose metabólica: 0 rim é a principal via de eliminação de ácidos não- voláteis. Com a diminuição da FG, ocorre limitação na excreção da carga de ácidos gerados, resultando em acidose metabólica compensada (assintomática) ou descompensada. ${ }^{30,31}$ A correção da acidose metabólica deve ser realizada com bicarbonato de sódio oral, de maneira a manter o bicarbonato sérico (dosado em sangue venoso) acima de $22 \mathrm{mEq} / \mathrm{L}$. Este procedimento parece se associar com menor velocidade de queda da FG. ${ }^{32}$

Dislipidemia: A DRC evolui com alta prevalência de alterações do metabolismo dos lipídeos. Consequentemente, é importante rastrear, avaliar e tratar as alterações do colesterol total, HDL-colesterol, LDL-colesterol e triglicerídes, mesmo nos estágios iniciais da DRC. Não existem, entretanto, evidências definitivas do benefício do tratamento da dislipidemia nos pacientes com doença renal, pois a maioria dos grandes estudos realizados até o momento excluíram portadores de DRC. Como consequência, são adotadas para os pacientes com DRC as mesmas recomendações aplicadas para a população geral: $2,3,4,5$

- O tratamento com estatina deve ser iniciado em todo o paciente com DRC estágios 1 a 3, deve ser iniciado com doses baixas e aumentar de acordo com a necessidade, como proposto nas recomendações para pacientes sem comprometimento renal.

- O tratamento com estatina em pacientes com DRC estágio quatro objetiva manter a LDL-colesterol $<100 \mathrm{mg} / \mathrm{dL}$.

- Os fibratos são recomendados para os pacientes com hipertrigliceridemia (após ajustes dietéticos e prática de exercícios). Deve-se evitar a associação de fibrato com estatina, devido ao risco de rabdomiólise e comprometimento da função hepática.

Diabetes: O Diabetes mellitus é a causa mais frequente de DRC no mundo e já é a segunda etiologia mais comum entre os pacientes em diálise no Brasil. ${ }^{1,33}$ Além disso, os diabéticos apresentam risco aumentado de eventos cardiovasculares. A hiperglicemia é um fator de risco independente para nefroesclerose diabética. ${ }^{34} \mathrm{~A}$ fisiopatologia da doença renal diabética é complexa, abrangendo fatores hemodinâmicos, concentração plasmática dos produtos finais de glicolisação avançada e disfunção endotelial entre outros. ${ }^{35} \mathrm{O}$ controle glicêmico nesses pacientes é um desafio, pois envolve orientação dietética 
complexa, aderência medicamentosa e limitação no uso dos hipoglicemiantes orais, particularmente nos estágios mais avançados da DRC (4 e 5). Recomenda-se manter a hemoglobina glicosilada em níveis $<7,0 \%$ e a glicemia pós-prandial $<140$ $\mathrm{mg} / \mathrm{dL} .{ }^{36}$ Seguem algumas orientações: ${ }^{5,36,37}$

- A metformina é recomendada para pacientes com Diabetes mellitus tipo 2 e DRC estágios 1 e 2 , nos quais a FG encontrase estável nos últimos três meses. Pode ser mantida em pacientes diabéticos tipo 2 e com DRC estágio 3 , desde que a FG mantenha-se estável. Devido ao perigo do acúmulo de ácido láctico, recomenda-se a interrupção do tratamento com metformina sempre que houver piora súbita da função renal, como, em: insuficiência cardíaca descompensada, tratamento com IECA ou BRAT 1 nos pacientes com doença das artérias renais, uso de anti-inflamatórios não-esteroides, estudos de imagem com contraste iodado e uso abusivo de diuréticos.

- Havendo contraindicação para o uso de metformina, deve-se escolher outro agente hipoglicemiante (incluindo a insulina), levando-se em consideração a situação da cada paciente em particular, o nível de FG e presença de comorbidades.

- Alertar o paciente para a possibilidade de hipoglicemia, orientar como reconhecê-la e tratá-la, particularmente naqueles em uso de insulina ou secretagogo de insulina.

Nos pacientes com DRC e diabetes, o tratamento com sulfonilureias de curta ação é mais indicado do que com as apresentações de longa ação.

Mudanças no estilo de vida: : $^{1,3-5}$ Os pacientes com DRC frequentemente exibem fatores de risco comuns às doenças cardiovasculares e ao Diabetes mellitus; em sendo assim, são mandatórias as seguintes medidas:

- Interromper o tabagismo, objetivando diminuir a progressão da DRC e reduzir os riscos cardiovasculares;

Adequar o peso corporal de maneira a manter o índice de massa corporal entre 18,5 e $24,9 \mathrm{~kg} / \mathrm{m}^{2}$ e a circunferência abdominal $<102 \mathrm{~cm}$ nos homens e $<88 \mathrm{~cm}$ nas mulheres;

- Reduzir ou interromper o consumo de álcool, objetivando melhor controle da pressão arterial nos hipertensos;

- Praticar exercícios diariamente (caminhada, jogging, bicicleta ou natação), por pelo menos 30 a 60 minutos para os pacientes com DRC e sem contraindicação médica;

- Controlar a ingestão de sal, que não deve ultrapassar 6 g/ dia, prescrever dieta individualizada de acordo com a recomendação médica.

Preparo para a terapia renal substitutiva: Os pacientes no estágio 5 da DRC devem ser preparados com antecipação para iniciar a terapia renal substitutiva, evitando-se procedimentos de urgência. Para tanto, recomenda-se que sejam tomadas as seguintes medidas ${ }^{2,5}$ : (1) vacinação contra o vírus da hepatite $B$ (que pode ser realizada em qualquer estágio da DRC); (2) suporte psicológico ao paciente e seus familiares; (3) suporte social; (4) oportunidade de discussão com o paciente e seus familiares sobre as modalidades de terapia renal substitutiva (tipo de diálise ou transplante) para que este possa fazer a sua escolha.

Por fim, chamamos a atenção de que diagnóstico e tratamento precoces são a melhor forma de prevenir a progressão da DRC rumo à perda irreversível de função, particularmente se todos os médicos atentarem para essa possibilidade diagnóstica. 0 nãonefrologista tem papel central na identificação e prevenção da doença, bem como no encaminhamento destes pacientes, especialmente nos grupos de risco já descritos. Vale menção especial a importância da pesquisa da microalbuminúria em diabéticos para prevenção da instalação e progressão da nefropatia diabética.

\section{Conflito de interesse: não há}

\section{SUMmARY}

\section{Chronic Kidney diseases: common and haRmful, but ALSO PREVEN-} TABLE AND TREATABLE

Chronic kidney disease (CKD) is a public health problem worldwide. In Brazil incidence and prevalence of end stage renal failure are increasing; prognosis is still poor and costs of disease treatment are very high. Regardless of the etiology, main outcomes in patients with CKD are its complications (anemia, metabolic acidosis, malnutrition and alteration in mineral metabolism), death (mainly due to cardiovascular causes) and loss of renal function. Recent studies indicate that these outcomes may be postponed with specific treatment if the CKD is diagnosed early and renoprotective and cardioprotective measures are implemented early in the course of the disease. The current definition and staging of CKD, as well as the discussion of the main preventive measures are addressed in this review. [Rev Assoc Med Bras 2010; 56(2): 248-53]

KEY words: Kidney diseases. Diabetes mellitus. Anemia. Proteinuria. Albuminuria. Glomerular filtration rate.

\section{REFERÊNCIAS}

1. K/DOQI clinical practice guidelines for chronic kidney disease: evaluation, classification and stratification. Am J Kidney Dis. 2002;39(Suppl 2):S1-S246.

2. Sesso R, Lopes, AA, Thomé AS, Bevilacqua, JL, Romão Junior JE, Lugon JR. elatório do Censo Brasileiro de Diálise, 2008 Relatório do Censo Brasileiro de Diálise, 2008 J Bras Nefrol. 2008;30:233-8.

3. Diretrizes Brasileiras de Doença Renal Crônica. J Bras Nefrol. 2004;26(Supl 1):S1-S49.

4. The National Collaborating Centre for Chronic Conditions. Chronic kidney disease. National clinical guideline for early identification and management in adults in primary and secondary care. Available from: http://www.rcplondon. ac.uk.

5. Levin A, Hemmelgarn B, Culleton B, Tobe S, McFarlane P, Ruzicka M, et al. Guidelines for the management of chronic diseases. CMAJ. 2008;179:115462. Available from: http://www.cmaj.ca/cgi/content/full/179/11/1154/DC1.

6. Davies DF, Shock NW. Age changes in glomerular filtration rate, effective renal plasma flow, and tubular excretory capacity in adult males. J Clin Invest. $1950 ; 29: 496-507$

7. Lindeman RD, Tobin J, Shock NW. Longitudinal studies on the rate of decline in renal function with age. J Am Geriatr Soc.1985;33:278-85.

8. Rowe JW, Andres R, Tobin JD, Norris AH, Shock NW. The effect of age on creatinine clearance in men: a cross-sectional and longitudinal study. J Gerontol.1976;31:155-63.

9. Fried LP, Kronmal RA, Newman AB, Bild DE, Mittelmark MB, Polak JF, et al. Risk factors for 5-year mortality in older adults: the Cardiovascular Health Study. JAMA 1998; 279:585-92.

10. Shlipak MG, Fried LF, Crump C, Bleyer AJ, Manolio TA, Tracy RP, et al. Cardiovascular disease risk status in elderly persons with renal insufficiency. Kidney Int. 2002;62:997-1004.

11. Manjunath G, Tighioaurt H, Coresh J, Macleod B, Salem DN, Griffith JL, et al. Level of kidney function as a risk factor for cardiovascular outcomes in the elderly. Kidney Int 2003;63:1121-9.

12. Coresh J, Astor BC, Greene T, Eknoyan G, Levey AS. Prevalence of chronic kidney disease and decreased kidney function in the adult US population: Third National Health and Nutrition Examination Survey. Am J Kidney Dis. 2003;41:1-12

13. Cockcroft DW, Gault MH. Prediction of creatinine clearance from serum creatinine. Nephron.1976;16:31-41.

14. Levey AS, Bosch JP, Lewis JB, Greene T, Rogers N, Roth D. A more accurate method to estimate glomerular filtration rate from serum creatinine: a new 
DOENÇA RENAL CRÔNICA: FREQUENTE E GRAVE, MAS TAMBÉM PREVENÍVEL E TRATÁVEL

prediction equation. Modification of Diet in Renal Disease Study Group. Ann Intern Med.1999;130:461-70.

15. Nóbrega AM, Gomes CP, Lemos CC, Bregman R. Is it possible to use modification of diet in renal disease (MDRD) equation in a Brazilian population? J Nephrol. 2006;19:196-9.

17. Bastos RMR, Bastos MG. Tabela de cálculo imediato de filtração glomerular. J Bras Nefrol. 2005;27:40-42.

18. Iseki K. Chronic kidney disease in Japan from early predictions to current facts. Nephron Clin Pract. 2008;110:268-72.

19. Culleton B. Introduction to the Canadian Clinical Practice Guidelines. J Am Soc Nephrol. 2006;17:S1-3.

20. Palmer BF. Renal dysfunction complicating the treatment of hyperensio. N Eng J Med. 2002;347:1256-61.

21. Remuzzi G, Bertani T. Pathophysiology of progressive nephropathies. N Engl J Med. 1998;339:1448-56.

22. Jafar TH, Stark PC, Schmid CH, Landa M, Maschio G, Jong PE, et al. Progression of chronic kidney disease: the role of blood pressure control, proteinuria, and angiotensin-converting enzyme inhibition: a patient level meta-analysis. Ann Intern Med. 2003;139:244-52.

23. Vaziri ND. Anemia and anemia correction: surrogate markers or causes of morbidity in chronic kidney disease? Nature Clin Pract Nephrol. 2008;4:436-45.

24. Nangaku M, Eckardt KU. Pathogenesis of renal anemia. Semin Nephrol. 2006; 26:261-8

25.Post JB, Wilkes BM, Michelis MF. Iron deficiency in patients with chronic kidney disease: potential role for intravenous iron therapy independent of erythropoietin. Int Urol Nephrol. 2006;38:719-23.

26. National Kidney Foundation: KDOQI Clinical Practice Guideline and Clinical Practice Recommendations for Anemia in Chronic Kidney Disease: 2007 Update of hemoglobin target. Am J Kidney Dis. 2007;50:471-530.

27. Diretriz para o Tratamento da Anemia no Paciente com Doença Renal Crônica. J Bras Nefrol. 2007;29(Supl 4):1-32.

28. Novaka JE, Szczech LA. Triumph and tragedy: anemia management in chronic kidney disease. Cur Opin Nephrol Hypertens. 2008;17:580-8.
29. K/DOQI Clinical Practice Guidelines for Managing Dyslipidemias in Chronic Kidney Disease. Am J Kidney Dis. 2003;41(Suppl 3):S39-S58.

30. Alpern RJ, Sakhaee K. The clinical spectrum of chronic metabolic acidosis: homeostatic mechanisms produce significant morbidity. Am J Kidney Dis. 1997;29:291-302.

31. OhMS. New perspectiveson acid-basebalance. Semin Dial. 2000;13:212-219.

32. Brito-Ashurst I, Varagunum M, Raftery MJ, Yoqoob MM. Bicarbonate supplementation slows progression of CKD and improves nutritional status. J Am Soc Nephrol. 2009;20:2075-2084.

33. Excerpts From the United States Renal Data System 2008 Annual Data Report Atlas of chronic kidney disease \& end-stage renal disease in the United States. Am J Kidney Dis. 2009;53(Suppl 1):S1-S374.

34. The effect of intensive treatment of diabetes on the development and progression of long-term complications in insulin-dependent diabetes mellitus, The Diabetes Control and Complication Trial Research Group. N Engl J Med. 1993;329:977-86.

35. Cooper M. Pathogenesis, prevention, and treatment of diabetic nephropathy. Lancet. 1998;352:213-9.

36. American Diabetes Association. Standards of medical care for patients with diabetes mellitus. Diabetes Care. 2003;26(Suppl 1):S33-50.

37. KDOQI Clinical Practice Guidelines and Clinical Practice Recommendations for Diabetes and Chronic Kidney Disease. Am J Kidney Dis. 2007;49(Suppl 2):S1-S182

Artigo recebido: 28/09/09 Aceito para publicação: 8/11/09 